\title{
Article \\ Design of Oral Sustained-Release Pellets by Modeling and Simulation Approach to Improve Compliance for Repurposing Sobrerol
}

\author{
Chu-Hsun Lu ${ }^{1,2, *}$, Yu-Feng Huang ${ }^{1}$ and I-Ming Chu ${ }^{2, *(D)}$ \\ 1 Biomedical Technology and Device Research Laboratories, Industrial Technology Research Institute, \\ Hsinchu 30011, Taiwan; markhuang@itri.org.tw \\ 2 Department of Chemical Engineering, National Tsing Hua University, Hsinchu 30013, Taiwan \\ * Correspondence: chuhsun@itri.org.tw (C.-H.L.); imchu@che.nthu.edu.tw (I.-M.C.)
}

check for

updates

Citation: Lu, C.-H.; Huang, Y.-F.;

Chu, I.-M. Design of Oral

Sustained-Release Pellets by

Modeling and Simulation Approach

to Improve Compliance for

Repurposing Sobrerol. Pharmaceutics

2022, 14, 167. https://doi.org/

10.3390/pharmaceutics14010167

Academic Editors: Luciana Scotti and

Anne Rodallec

Received: 8 December 2021

Accepted: 7 January 2022

Published: 11 January 2022

Publisher's Note: MDPI stays neutral with regard to jurisdictional claims in published maps and institutional affiliations.

Copyright: (c) 2022 by the authors. Licensee MDPI, Basel, Switzerland. This article is an open access article distributed under the terms and conditions of the Creative Commons Attribution (CC BY) license (https:// creativecommons.org/licenses/by/ $4.0 /)$.

\begin{abstract}
Sobrerol, an oral mucolytic agent, in a recent study showed promise for treating multiple sclerosis. A human equivalent dose of $486 \mathrm{mg}$ of sobrerol administered thrice daily (i.e., $1459 \mathrm{mg}$ of daily dose) demonstrated the highest therapeutic efficacy for repurposing use, which also points out the poor compliance of administration. In this study, oral sustained-release pellets of sobrerol were successfully developed with evaluated manufacturing conditions and drug release kinetics. For design of the target drug product, we used a modeling and simulation approach to establish a predictive model of oral pharmacokinetic profile, by exploring the characteristics and correlations corresponding to the pharmacokinetics and pharmacodynamics of sobrerol, such as absorption lag time $(0.18 \mathrm{~h})$, time-scaling in vitro-in vivo correlation $\left(t_{\text {in-vitro }}=0.494 t_{\text {in-vivo }}-0.0904\right)$, gastrointestinal transit time $(8 \mathrm{~h})$, minimum effective concentration $(1.61 \mu \mathrm{g} / \mathrm{mL})$, and duration of action $(12.8 \mathrm{~h})$. Results showed that the frequency of administration and the daily dose remarkably reduced by $33.3 \%$ (i.e., from thrice to twice daily) and $22.8 \%$, respectively, which indicates that this prototype approach can be adopted for rapidly developing a modified-release dosage form of sobrerol, with improvement of compliance of administration and therapeutic efficacy.
\end{abstract}

Keywords: sobrerol; multiple sclerosis; sustained-release; pellets; modeling and simulation; dissolution; pharmacokinetics; pharmacodynamics; IVIVC

\section{Introduction}

Sobrerol, developed in the 1970s as a mucolytic agent, is widely used to treat acute or chronic respiratory diseases caused by increased bronchial mucus and obstruction [1] The recommended dose of sobrerol was $400 \mathrm{mg}$ daily for adults, the longest treatment period was 3 months with a daily dose of $600 \mathrm{mg}$, and the maximum daily dose was $900 \mathrm{mg}$ for 10 consecutive days. Sobrerol is considered a relatively safe drug with no substantial side effects when administered under guidance [2-4]. In addition to respiratory diseases, sobrerol has recently been studied to treat multiple sclerosis, an autoimmune neurological disease [5]. The experimental autoimmune encephalomyelitis (EAE) animal model exhibiting inflammatory demyelination and axonal damage was adopted in the study on multiple sclerosis treatment [6,7], and sobrerol could effectively alleviate EAE symptoms at a dose of $100 \mathrm{mg} / \mathrm{kg}$ thrice daily, which is equivalent to the positive control dimethyl fumarate at a dose of $100 \mathrm{mg} / \mathrm{kg}$ twice daily. Dimethyl fumarate is the first-line oral drug currently used for treating multiple sclerosis, and its most common adverse events are flushing, diarrhea, abdominal pain, nausea, vomiting, and lymphocyte reduction [8,9]. Compared with dimethyl fumarate, sobrerol has the potential to be safer in treatment. However, the human equivalent dose converted from the animal dose is $486 \mathrm{mg}$ thrice daily (i.e., $1459 \mathrm{mg}$ of daily dose), which considerably exceeds the current therapeutic dose used. 
In this study, we developed oral sobrerol sustained-release (SR) pellets to improve compliance for repurposing indications. SR pellets are usually pseudospherical (0.5-1.5 mm in diameter) with a smooth surface, relatively high density, and excellent flowability. SR pellets dosage form is comprised of many small discrete drug delivery units, which can then be filled into capsules or sachets for oral consumption [10-13]. SR pellets not only have flexible release profiles but can also reduce the risk of dose dumping compared with larger single-unit dosage forms [14-16], thus demonstrating the advantages of long-term efficacy and safety.

The Biopharmaceutics Classification System (BCS) is a systematic classification based on the water solubility and intestinal permeability of drugs (as shown in Table S1 of Supplementary Material) [17]. When combined with in vitro dissolution characteristics, this system can be used to determine how fast and how much of a drug is absorbed in the body. To shorten the time and reduce the cost during the development of drug products, in vitro-in vivo correlations (IVIVCs) are often used to express the characteristics of drug products; that is, they accurately predict the correlation of the in vivo pharmacokinetic profiles of drugs with the in vitro dissolution data. If the drugs with a property of high permeability (i.e., BCS class I or II) are stable in the gastrointestinal tract and dissolution is the rate-limiting step during drug absorption, the IVIVCs are expectedly established [18,19]. Therefore, sobrerol can be classified as a BCS class I drug due to high water solubility and intestinal permeability $[20,21]$, which is supported to follow the model of IVIVC, even more in the case of modified-release.

As depicted in Scheme 1, we developed pellet dosage forms and evaluated manufacturing conditions and drug release kinetics for various pellets. Subsequently, we predicted the oral pharmacokinetic profiles of pellet dosage forms by adopting modeling and simulation approaches [22,23]. We constructed a predictive model by exploring the characteristics and correlations corresponding to the pharmacokinetics and pharmacodynamics of sobrerol. Finally, we used the constructed predictive model to design the formula and specification of the target drug product of sobrerol to improve compliance of administration on repurposing treatment.

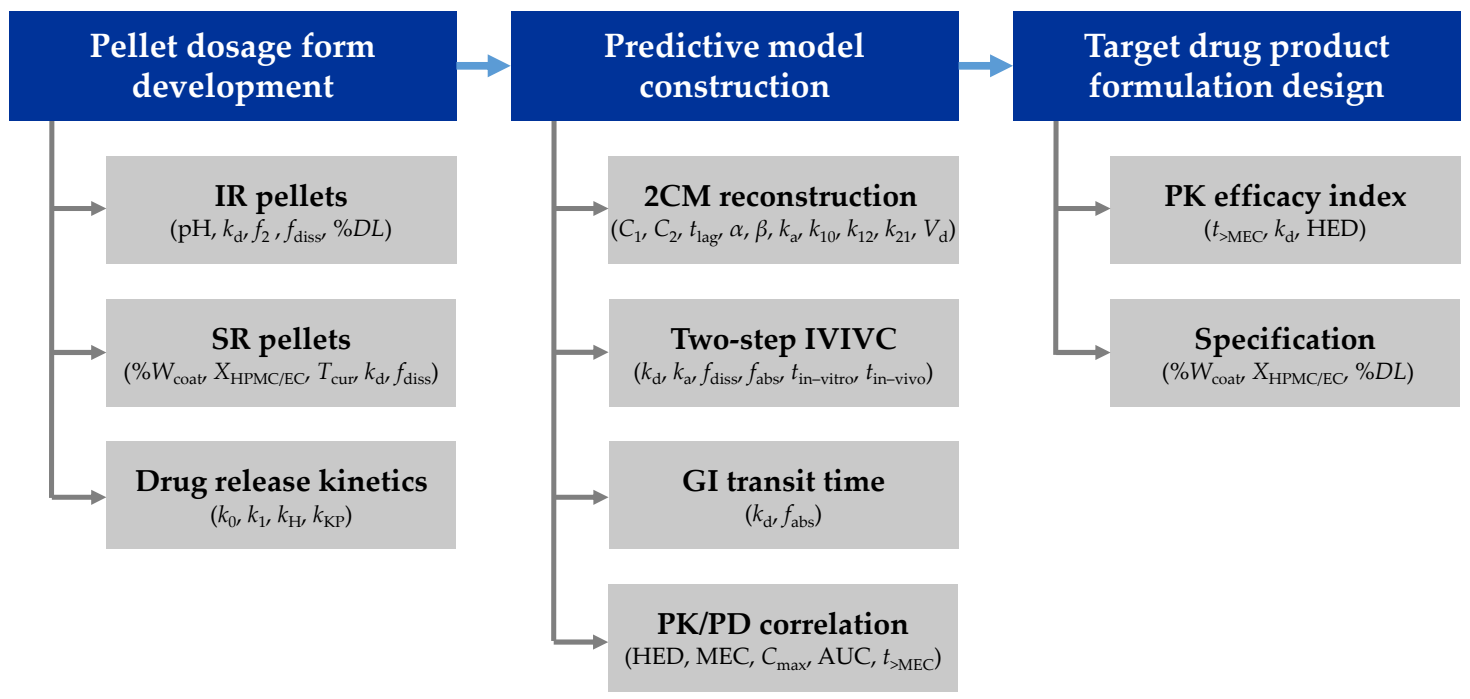

Abbreviations:

IR, immediate-release; SR: sustained-release; 2CM, two-compartment model; IVIVC: in vitro-in vivo correlation; GI: gastrointestinal; PK: pharmacokinetics; PD: pharmacodynamics; $k_{\mathrm{d}}$, the first-order constant of dissolution; $f_{2}$, the similarity factor; $f_{\text {diss }}$ the fraction of the drug dissolved; $\% D L$, the drug loading; $\% W_{\text {coat }}$ the coating weight relative to IR pellets (DoE); $X_{\mathrm{HPMC} / \mathrm{EC}} \mathrm{HPMC/EC} \mathrm{composition} \mathrm{ratio} \mathrm{(DoE);} T_{\text {cur }}$ the curing temperature (DoE); $k_{0}$, the release constant of the zero-order model; $k_{1}$, the release constant of first-order model; $k_{\mathrm{H}}$, the release constant of the Higuchi model; $k_{\mathrm{KP}}$, the release constant of the Korsmeyer-Peppas model; $C_{1}$, the concentrations in the central compartment; $C_{2}$, the concentrations in the peripheral compartment; $t_{\text {lag, }}$ the lag time; $\alpha$, the distribution rate constant; $\beta$, the elimination rate constant; $k_{a}$, the firstorder rate constant for absorption through oral administration; $k_{10}$, the first-order rate constant for elimination; $k_{12}$, the first-order rate constant for distribution from the central to peripheral compartment; $k_{21}$, the first-order rate constant for distribution from the peripheral to central compartment; $V_{\mathrm{d}}$, the volume of distribution; $f_{\mathrm{abs}}$ the fraction of the drug absorbed; $t_{\text {in-vitro }}$ the time of in vitro dissolution; $t_{\text {in-vivo }}$ the time of in vivo absorption; HED, the human equivalent dose; MEC, the minimum effective concentration; $C_{\max }$ the peak plasma concentration; AUC, the area under the curve; $t_{\mathrm{SEC}}$ the duration of action.

Scheme 1. A flowchart for developing oral sobrerol pellet dosage form to improve compliance in repurposing treatment. 


\section{Materials and Methods}

\subsection{Materials}

Sobrerol was purchased from DaeHe Biopharma Co., Ltd. (Gyeonggi-do, Korea). Ethanol and analytical-grade methanol were obtained from Echo Chemical Co., Ltd. (Miaoli, Taiwan). Microcrystalline cellulose (MCC) was obtained from Wei Ming Pharmaceutical Mfg. Co., Ltd. (Taipei, Taiwan). Ethyl cellulose (EC) and hydroxypropyl methyl cellulose (HPMC) were obtained from Ashland Global Specialty Chemicals Inc. (Ashland, KY, USA).

\subsection{Manufacturing}

\subsubsection{IR Pellets}

Considering that a higher dose is required for repurposing sobrerol, we used the extrusion-spheronization process that can produce high-drug-loaded dosage forms to prepare sobrerol immediate-release (IR) pellets [24,25]. Subsequently, $84 \mathrm{~g}$ of sobrerol, $36 \mathrm{~g}$ of MCC, and an appropriate amount of water were thoroughly mixed using a mixer (TK9517, Heas Technology Co., Ltd., New Taipei, Taiwan). The pellets were prepared into a strip shape by using an extruder (SY-BG-120, Shang-Yuh Machine Co., Ltd., New Taipei, Taiwan) at a speed of $40 \mathrm{rpm}$. Spherical pellets were produced using a spheronizer (SY-SM40C, Shang-Yuh Machine Co., Ltd., New Taipei, Taiwan) at a speed of $1000 \mathrm{rpm}$ for $1 \mathrm{~min}$. The pellets were finally dried in a hot-air oven (ED-4, Rei Hsiung Enterprise Co., Ltd., New Taipei, Taiwan) at $60{ }^{\circ} \mathrm{C}$ for $3 \mathrm{~h}$ to obtain IR pellets with $70 \%$ drug loading.

\subsubsection{SR Pellets}

SR pellets were prepared by spraying medical polymers on the IR pellets to form a coating layer. The coating layer was composed of a hydrophobic polymer (EC) and a hydrophilic polymer (HPMC). The formula and preparation conditions were examined using a 3-factor, 3-level design of experiment $\left(3^{3} \mathrm{DoE}\right)$. A 13-experiment-matrix was generated using Minitab 18 (Minitab LLC., State College, PA, USA) with three factors, namely, coating weight relative to IR pellets ( $\% W_{\text {coat }}$, Factor A), HPMC $/ \mathrm{EC}$ composition ratio $\left(X_{\mathrm{HPMC} / \mathrm{EC}}\right.$, Factor B), and curing temperature ( $T_{\text {cur }}$, Factor $\left.C\right)$. Each factor had three levels and was coded from low to high with values of $-1,0$, and 1 , respectively, and the response indicated the first-order constant of dissolution $\left(k_{\mathrm{d}}\right.$ in unit of $\left.\mathrm{h}^{-1}\right)$. The $3^{3}$ DoE matrix of sobrerol SR pellets is presented in Table 1, and actual weights of the components are shown in Table S2 of Supplementary Material.

Table 1. The $3^{3}$ DoE matrix of sobrerol SR pellets for a total of 13 experiments.

\begin{tabular}{cccc}
\hline Code & $\begin{array}{r}\text { Factor } \mathbf{A}^{\mathbf{1}} \\
\mathbf{\%} \boldsymbol{W}_{\text {coat }}\end{array}$ & $\begin{array}{c}\text { Factor B } \\
\boldsymbol{X}_{\text {HPMC/EC }}\end{array}$ & $\begin{array}{c}\text { Factor C }^{\mathbf{3}} \\
\boldsymbol{T}_{\text {cur }}\end{array}$ \\
\hline SR-1 & 0 & +1 & +1 \\
SR-2 & +1 & -1 & 0 \\
SR-3 & +1 & 0 & +1 \\
SR-4 & -1 & -1 & 0 \\
SR-5 & 0 & +1 & -1 \\
SR-6 & -1 & +1 & 0 \\
SR-7 & 0 & 0 & 0 \\
SR-8 & +1 & 0 & -1 \\
SR-9 & 0 & -1 & -1 \\
SR-10 & +1 & +1 & 0 \\
SR-11 & 0 & -1 & +1 \\
SR-12 & -1 & 0 & +1 \\
SR-13 & -1 & 0 & -1 \\
\hline
\end{tabular}

1 Values of level of $-1,0,1$ corresponding to Factor A: 6.9\%, 8.3\%, and $9.7 \%$. ${ }^{2}$ Values of level of $-1,0,1$ corresponding to Factor B: $0.20,0.25$, and $0.30 .{ }^{3}$ Values of level of $-1,0,1$ corresponding to Factor $\mathrm{C}: 50^{\circ} \mathrm{C}, 60^{\circ} \mathrm{C}$, and $70{ }^{\circ} \mathrm{C}$. 
SR pellets were prepared using the following steps. Various compositions of HPMC and EC were dissolved in $50 \%(v / v)$ of ethanol to prepare a spraying solution. Subsequently, $180 \mathrm{~g}$ of IR pellets (diameter from 500 to $710 \mu \mathrm{m}$ ) were coated with the spraying solution using a fluidized bed granulator (FGA-16, Ohkawara Kakohki Co., Ltd., Kanagawa-ken, Japan) at a feed rate of $0.9-1.4 \mathrm{~g} / \mathrm{min}$; other operating conditions were as follows: air pressure, $1.0-1.6 \mathrm{~kg} / \mathrm{cm}^{2}$; inlet air temperature, $28-33{ }^{\circ} \mathrm{C}$; and exhaust air temperature, 20-23 ${ }^{\circ} \mathrm{C}$. Finally, the coated pellets were cured in an oven (DO45, Dogger Scientific Co., Ltd., New Taipei, Taiwan) at a set temperature for 6 h. Finally, 13 types of sobrerol SR pellets were obtained.

\subsection{Characterization}

\subsubsection{Scanning Electron Microscopy (SEM)}

The morphological properties of IR and SR pellets were investigated using an SEM (SU8010, Hitachi Ltd., Tokyo, Japan). The cross-sectional view of the pellet was obtained by cutting the SR pellet with a blade. SEM images were acquired at an accelerated voltage of $10 \mathrm{kV}$ under different magnifications.

\subsubsection{High-Performance Liquid Chromatography (HPLC)}

Sobrerol was assayed through HPLC (Waters Alliance 2695, Waters Corporation, Milford, MA, USA) with C18 column (Inertsil ODS-2, GL Sciences Inc., Tokyo, Japan). Samples were diluted with $50 \%(v / v)$ of methanol from $100 \mu \mathrm{L}$ to $1 \mathrm{~mL}$ and filtered through $0.45-\mu \mathrm{m}$ filtration membranes. The operating conditions were as follows: detection wavelength of $\mathrm{UV}, 210 \mathrm{~nm}$; flow rate, $0.8 \mathrm{~mL} / \mathrm{min}$; injection volume, $10 \mu \mathrm{L}$; and mobile phase, $50 \%(v / v)$ of methanol [26]. Verification of a calibration curve (the $R^{2}$ is not $<0.995$ ) and reproducibility (the relative standard deviation of peak areas is not $>2.5 \%$ in 5 sets of injection) is conducted for system suitability before samples assayed.

\subsubsection{Dissolution Test}

The dissolution test was performed using USP apparatus 1 (the basket method). We poured $1000 \mathrm{~mL}$ of dissolution medium (i.e., pure water; $\mathrm{pH} 1.2,4.5$, or 6.8 of buffer solutions) into the vessel of the dissolution tester (DT-6, Shin-Kwang Precision Industry, New Taipei, Taiwan). Subsequently, we dropped IR pellets containing 100-130 mg of sobrerol into the basket placed in the dissolution medium, with stirring at $75 \mathrm{rpm}$. The whole process was performed at $37 \pm 0.5^{\circ} \mathrm{C}$. Sampling was performed at 5, 10, 15, 30, 45, $60,75,90$, and $120 \mathrm{~min}$. For SR pellets, each type was sampled for evaluation at 0.5, 1, 2, 4, 6, and $8 \mathrm{~h}$, and the dissolution medium used was pure water. Except for the aforementioned parameters, other operating conditions were the same as those used for IR pellets.

\subsubsection{The Drug Release Mechanism}

The fraction of the drug dissolved $\left(f_{\text {diss }}\right)$ was calculated for each pellet dosage form. The calculation of the similarity factor $\left(f_{2}\right)$ for IR pellets is shown in Section S2 of Supplementary Material [27]. The drug release mechanism for SR pellets was fitted using four kinetic models (zero-order, first-order, Higuchi, and Korsmeyer-Peppas) as shown in Table S3 of Supplementary Material [28,29].

\subsection{Establishing the Predictive Model}

\subsubsection{Reconstruction of the Sobrerol Pharmacokinetic Model}

According to a previous study, the plasma concentrations of a single oral dose of $300 \mathrm{mg}$ of sobrerol at $0.25,0.5,1,2,3,4,6$, and $8 \mathrm{~h}$ were 1.01, 2.82, 3.77, 2.02, 1.41, 1.05, 0.63 , and $0.37 \mu \mathrm{g} / \mathrm{mL}$, respectively [20]. Similarly, using the oral two-compartment model derived from mass balance methods (as depicted in Scheme S1 of Supplementary Material) and considering the lag time of drug absorption, pharmacokinetic parameters were recon- 
structed using WinNonlin 4.1 (Certara Inc., Princeton, NJ, USA). The oral two-compartment model is described by the following equations [30]:

$$
\begin{gathered}
C_{1}(t)=\frac{k_{\mathrm{a}} F D}{V_{\mathrm{d}}}\left(\frac{\left(k_{21}-\alpha\right)}{(\beta-\alpha)\left(k_{\mathrm{a}}-\alpha\right)} e^{-\alpha\left(t-t_{\mathrm{lag}}\right)}+\frac{\left(k_{21}-\beta\right)}{\left(k_{\mathrm{a}}-\beta\right)(\alpha-\beta)} e^{-\beta\left(t-t_{\operatorname{lag}}\right)}+\right. \\
\left.\frac{\left(k_{21}-k_{\mathrm{a}}\right)}{\left(\alpha-k_{\mathrm{a}}\right)\left(\beta-k_{\mathrm{a}}\right)} e^{-k_{\mathrm{a}}\left(t-t_{\mathrm{lag}}\right)}\right) \\
C_{2}(t)=\frac{k_{\mathrm{a}} k_{12} F D}{V_{\mathrm{d}}} \quad\left(\frac{1}{(\beta-\alpha)\left(k_{\mathrm{a}}-\alpha\right)} e^{-\alpha\left(t-t_{\operatorname{lag}}\right)}\right. \\
+\frac{1}{\left(k_{\mathrm{a}}-\beta\right)(\alpha-\beta)} e^{-\beta\left(t-t_{\mathrm{lag}}\right)} \\
\left.+\frac{1}{\left(\alpha-k_{\mathrm{a}}\right)\left(\beta-k_{\mathrm{a}}\right)} e^{-k_{\mathrm{a}}\left(t-t_{\mathrm{lag}}\right)}\right)
\end{gathered}
$$

where $C_{1}$ and $C_{2}(\mu \mathrm{g} / \mathrm{mL})$ are the concentrations in the central and peripheral compartments, respectively, at time $t, k_{\mathrm{a}}\left(\mathrm{h}^{-1}\right)$ is the first-order rate constant for absorption through oral administration, $F$ is bioavailability, $D(\mu \mathrm{g})$ is the dose, $V_{\mathrm{d}}(\mathrm{mL})$ is the volume of distribution, $k_{12}\left(\mathrm{~h}^{-1}\right)$ and $k_{21}\left(\mathrm{~h}^{-1}\right)$ are the first-order rate constants for distribution from the central to peripheral compartment and back, respectively, $k_{10}\left(\mathrm{~h}^{-1}\right)$ is the first-order rate constant for elimination, $\alpha\left(\mathrm{h}^{-1}\right)$ is the distribution rate constant, $\beta\left(\mathrm{h}^{-1}\right)$ is the elimination rate constant, $t(\mathrm{~h})$ is time, and $t_{\text {lag }}(\mathrm{h})$ is the lag time.

\subsubsection{Exploring the IVIVC of Sobrerol}

The two-step approach was used to construct a valid IVIVC of sobrerol [31,32]. In the first step, the pharmacokinetic profile shown in Section 2.4.1 was transformed to the in vivo fraction of the drug absorbed $\left(f_{\text {abs }}\right)$ by deconvolution, and a time-scaling IVIVC was explored by relating the times of in vitro dissolution $\left(t_{\text {in-vitro }}\right)$ and those of in vivo absorption $\left(t_{\text {in-vivo }}\right)$ at equivalent fractions. In the second step, the first-step $f_{\text {abs }}$ was reconverted by convolution to generate a pharmacokinetic profile that was comparable to the original one for model validation. Equations (3) and (4) were used for the deconvolution and calculation of $f_{\text {abs }}$, respectively, and Equation (5) was used for convolution to predict in vivo pharmacokinetic profiles [33], which are described as follows:

$$
\begin{gathered}
\Delta D(t)=\left\{\Delta C_{1}(t)+\left(k_{10} C_{1}(t)+k_{12} C_{1}(t)-k_{21} C_{2}(t)\right) \times \Delta t\right\} \times V_{\mathrm{d}} \\
f_{\mathrm{abs}}(t)=\frac{\sum \Delta D(t)}{D} \\
C_{1}(t)=\sum_{i=0}^{t}\left\{\frac{D \times \Delta f_{\mathrm{abs}}(i)}{V_{\mathrm{d}}}\left(\frac{\left(k_{21}-\alpha\right)}{(\beta-\alpha)} e^{-\alpha(t-i)}+\frac{\left(k_{21}-\beta\right)}{(\alpha-\beta)} e^{-\beta(t-i)}\right)\right\}
\end{gathered}
$$

where $\Delta D(\mu \mathrm{g})$ is the drug absorbed at time $t, \Delta C_{1}(\mu \mathrm{g} / \mathrm{mL})$ is the concentration change in the central compartment at time $t, f_{\mathrm{abs}}$ and $\Delta f_{\mathrm{abs}}$ are the accumulated and partial fraction absorbed, respectively, at time $t$, and $i$ (h) is the absorption onset time of the partial fraction absorbed.

\subsubsection{Consideration of the Gastrointestinal Transit Time}

The absorption process of oral drugs in the gastrointestinal tract is considerably complicated, and the small intestine is the major site of drug absorption. Therefore, the gastrointestinal transit time should be considered during the development of SR dosage forms to ensure the extent of the release and absorption of the drug. The gastric transit can range from 0 to $2 \mathrm{~h}$ in the fasting state, and the transit time in the small intestine can range from 2 to $6 \mathrm{~h} \mathrm{[34].} \mathrm{The} \mathrm{gastrointestinal} \mathrm{transit} \mathrm{time} \mathrm{is} \mathrm{estimated} \mathrm{to} \mathrm{be} 8 \mathrm{~h}$ for sobrerol pharmacokinetic profile simulation. The plasma concentration during absorption in $8 \mathrm{~h}$ follows Equation (1), and that after $8 \mathrm{~h}$ is ruled by the intravenous two-compartment pharmacokinetic model, which can be determined using Equation (6):

$$
C_{1}(t)=\frac{D}{V_{\mathrm{d}}}\left(\frac{\left(k_{21}-\alpha\right)}{(\beta-\alpha)} e^{-\alpha t}+\frac{\left(k_{21}-\beta\right)}{(\alpha-\beta)} e^{-\beta t}\right)
$$




\subsubsection{Finding the Pharmacokinetic Efficacy Index}

The human equivalent dose (HED) and expected therapeutic efficacy (inhibition of symptoms) of sobrerol for multiple sclerosis treatment were derived from EAE animal tests performed in a previous study (C57BL/ 6 mice with EAE induced by MOG peptides were administered sobrerol for 14 days after onset. ${ }^{* *} p<0.01$ and ${ }^{* * *} p<0.001$ compared with the results for vehicle determined using Student's $t$-test) [5]. On the last day after onset (i.e., the plateau phase), low to high doses were used in this study for vehicle (thrice daily), namely, $25 \mathrm{mg} / \mathrm{kg}$ (thrice daily), $30 \mathrm{mg} / \mathrm{kg}$ (once daily), $100 \mathrm{mg} / \mathrm{kg}$ (thrice daily), $150 \mathrm{mg} / \mathrm{kg}$ (twice daily), and $300 \mathrm{mg} / \mathrm{kg}$ (once daily), and the corresponding EAE scores were $4.00 \pm 0.22,3.70 \pm 0.15,3.38 \pm 0.27,2.36 \pm 0.27^{* *}, 2.55 \pm 0.27^{* *}$, and $2.78 \pm 0.25^{* * *}$, respectively. The HED conversion based on the body surface area is shown below [35]:

$$
\operatorname{HED}(\mathrm{mg})=\text { Animal dose }\left(\frac{\mathrm{mg}}{\mathrm{kg}}\right) \times \frac{\text { Animal } K_{\mathrm{m}}}{\operatorname{Human} K_{\mathrm{m}}} \times \text { human body weight }(\mathrm{kg})
$$

where animal $K_{\mathrm{m}}$ (mouse) and human $K_{\mathrm{m}}$ (adults) are 3 and 37, respectively, and the human body weight is considered $60 \mathrm{~kg}$ for adults. Subsequently, the HED was used to simulate pharmacokinetic profiles by using Equation (1), and the peak plasma concentration $\left(C_{\max }\right)$ of the highest HED with no significant difference was assumed to be the minimum effective concentration (MEC). Three pharmacokinetic efficacy indexes, namely, the $C_{\max }$, the area under the curve (AUC), and the duration of action $\left(t_{>\mathrm{MEC}}\right)$ of each pharmacokinetic profile, were calculated to determine the pharmacokinetics/pharmacodynamics correlation [36]. In this study, Excel 2016 (Microsoft Corporation, Redmond, WA, USA) was used for calculating data and drawing figures.

\section{Results and Discussion}

\subsection{Preparation of IR/SR Pellets and Dissolution Study}

Sobrerol IR pellets were formed in a matrix structure, and the drug loading was more than $70 \%$ by total weight. Sobrerol was dispersed in the interstitium of MCC, with a rough and fiber-scattering appearance (Figure 1a). SR pellets were produced from IR pellets with HPMC/EC coated on the surface, which formed a smooth and continuous coating layer with SR function (Figure 1b). In the cross-sectional view, the interstitium of SR pellets showed an irregular crystal arrangement and tiny pores, and a clear interface between the IR and SR coating layer could be observed (Figure 1c). The thickness of the SR coating layer was approximately $5-10 \mu \mathrm{m}$ (Figure 1d).

As shown in Figure S1 of Supplementary Material, the $f_{\text {diss }}$ of IR pellets in media with different $\mathrm{pH}$ values all exceeded $80 \%$ at $30 \mathrm{~min}$ and reached the plateau value of $95-100 \%$ at $60 \mathrm{~min}$. No degradation was observed, indicating that sobrerol was stable even under $\mathrm{pH}$ changes in the gastrointestinal tract and is thus suitable for establishing an IVIVC. The similarity $f_{2}$ of the dissolution curves of IR pellets was more than 50 (Table S4 of Supplementary Material), indicating a high similarity of dissolution between various $\mathrm{pH}$ media. Thus, water was chosen as the dissolution medium in the following study, and the first-order $k_{\mathrm{d}}$ of IR pellets was calculated as $4.99 \mathrm{~h}^{-1}$.

Figure 2 shows the result of the dissolution test for 13 types of SR pellets. Analysis of DoE indicated that Factor B $\left(X_{\mathrm{HPMC} / \mathrm{EC}}\right)$ exerted a critical effect on $S R$ pellets (i.e., the higher the $X_{\mathrm{HPMC} / \mathrm{EC}}$ is, the higher is the dissolution rate), and a strong interaction was noted between Factor A (\% $W_{\text {coat }}$ ) and Factor B. Moreover, the 13 fitting curves were well plotted. Among the kinetic models, the first-order model could most favorably explain the drug release of SR pellets in the $0-8$-h interval. The first-order $k_{\mathrm{d}}$ values calculated from high to low were $1.082 \mathrm{~h}^{-1}$ (SR-5), $0.978 \mathrm{~h}^{-1}$ (SR-10), $0.858 \mathrm{~h}^{-1}$ (SR-1), $0.393 \mathrm{~h}^{-1}$ (SR-6), $0.131 \mathrm{~h}^{-1}$ (SR-13), $0.115 \mathrm{~h}^{-1}$ (SR-12), $0.115 \mathrm{~h}^{-1}$ (SR-7), $0.096 \mathrm{~h}^{-1}$ (SR-3), $0.059 \mathrm{~h}^{-1}$ (SR-8), $0.048 \mathrm{~h}^{-1}$ (SR-4), $0.036 \mathrm{~h}^{-1}$ (SR-2), $0.036 \mathrm{~h}^{-1}$ (SR-9), and $0.029 \mathrm{~h}^{-1}$ (SR-11), respectively. Additional analytical results of the DoE are provided in Figure S2 of Supplementary Material, and 
an example for designing a drug product with the target $k_{\mathrm{d}}$ is discussed in Section 3.3.

Dissolution mechanisms are provided in Table S5 and Figure S3 of Supplementary Material.
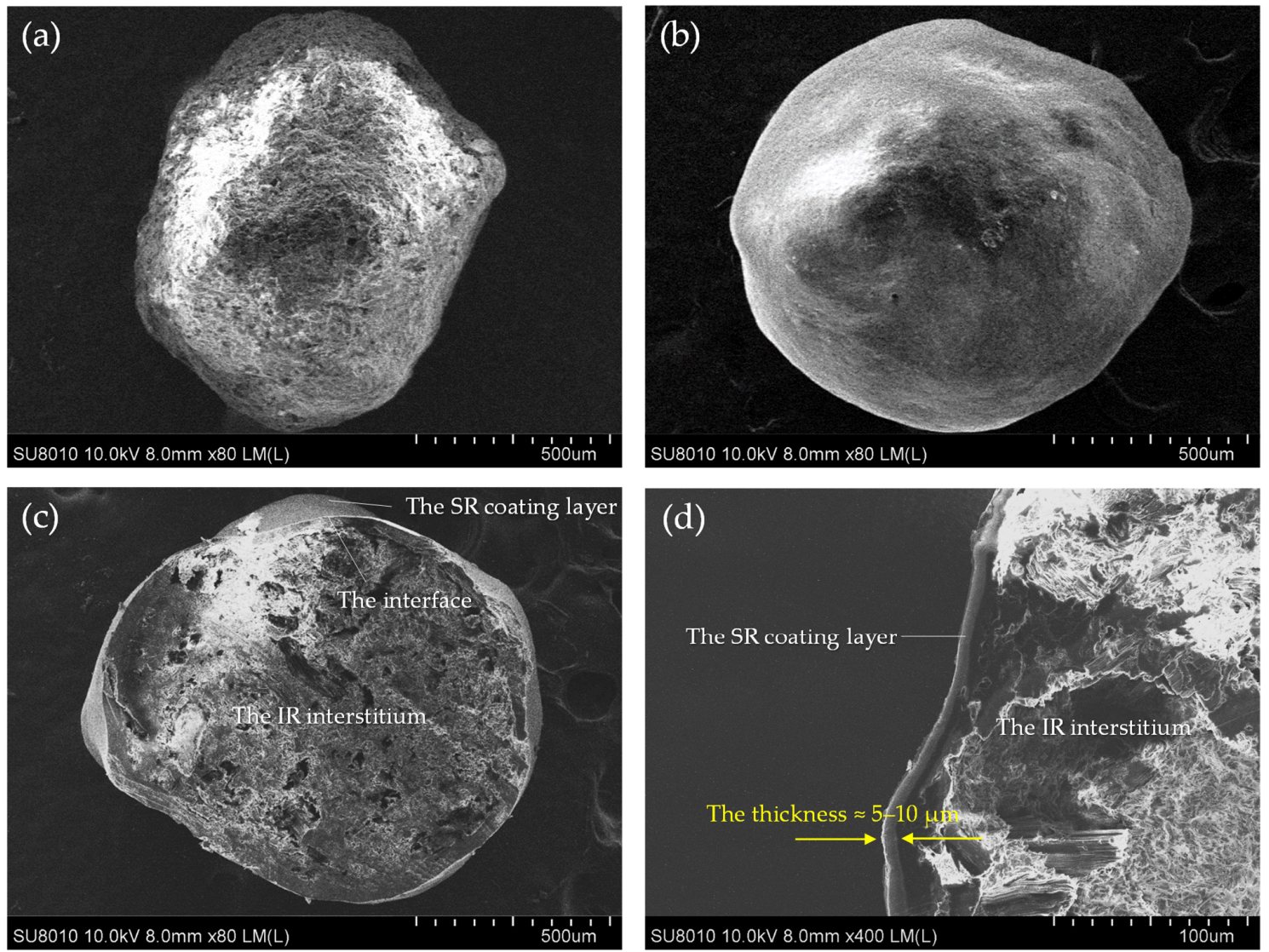

Figure 1. Morphological properties of IR and SR pellets determined using SEM: (a) the appearance of IR pellets; (b) the appearance of SR pellets; (c) the cross-sectional view of SR pellets; and (d) the estimation of the thickness of the SR coating layer to be 5-10 $\mu \mathrm{m}$.

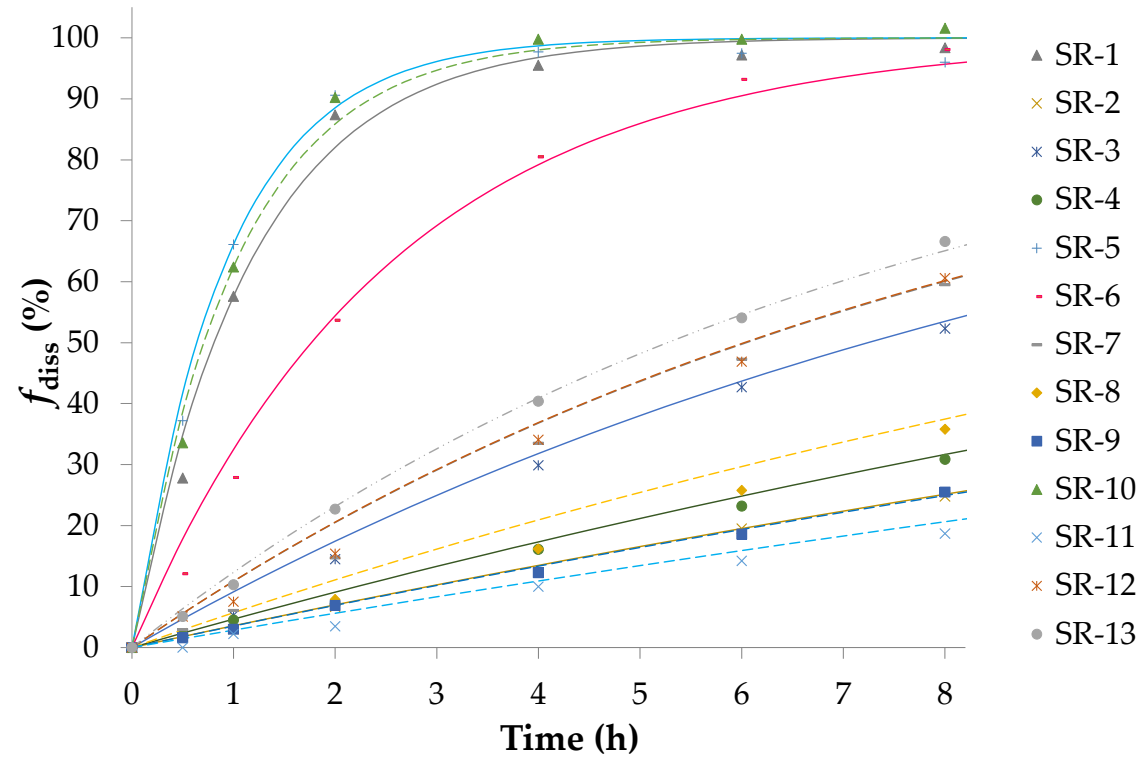

Figure 2. Dissolution tests of sobrerol SR pellets and the first-order fitting curves. The first-order model could most favorably explain the drug release of SR pellets in the 0-8-h interval. 


\subsection{Establishment of the Predictive Model}

3.2.1. Reconstruction of the Sobrerol Pharmacokinetic Model with Lag Time

A sobrerol two-compartment pharmacokinetic model associated with the lag time of drug absorption was established, with $V_{\mathrm{d}}$ of $44.94 \mathrm{~L} / \mathrm{h}, k_{12}$ of $0.82 \mathrm{~h}^{-1}, k_{21}$ of $0.95 \mathrm{~h}^{-1}, k_{\mathrm{a}}$ of $2.40 \mathrm{~h}^{-1}, \alpha$ of $2.08 \mathrm{~h}^{-1}, \beta$ of $0.26 \mathrm{~h}^{-1}$, and $t_{\text {lag }}$ of $0.18 \mathrm{~h}$. The aforementioned kinetic parameters were incorporated in Equations (1) and (2), and the simulated plasma concentrations over time are shown in Figure S4 of Supplementary Material. The fitting coefficients $\left(\mathrm{R}^{2}\right)$ calculated from the reconstructed model and a previous study [20] were 0.9796 and 0.9257 , respectively, indicating that the sobrerol pharmacokinetic model with lag time had more explanatory power.

\subsubsection{Time-Scaling IVIVC of Sobrerol}

The in vivo fraction of the drug absorbed $\left(f_{\text {abs }}\right)$, which was obtained by deconvolving the reconstructed sobrerol pharmacokinetic profile, is shown in Figure 3a. Sobrerol orally administered had an $f_{\text {abs }}$ of $>85 \%$ in $1 \mathrm{~h}$; thus, it could be classified as a fastabsorbing drug. A satisfactory linear regression of dissolution $t_{\text {in-vitro }}$ versus absorption $t_{\text {in-vivo }}$ is shown in Figure $3 \mathrm{~b}$, indicating that sobrerol had a favorable time-scaling IVIVC $\left(t_{\text {in-vitro }}=0.494 t_{\text {in-vivo }}-0.0904\right)$. Based on this correlation, a corresponding $f_{\text {abs }}$ at a certain time could be predicted through in vitro dissolution (e.g., $f_{\text {diss }}$ at $0.4 \mathrm{~h}$ is equivalent to $f_{\text {abs }}$ at $1.0 \mathrm{~h}$ ). In the second step, as shown in Figure S5 of Supplementary Material, a reconverted pharmacokinetic profile obtained by convolution fitted well to the original one, showing validation of the IVIVC model. A relationship between $k_{\mathrm{d}}$ and $k_{\mathrm{a}}\left(k_{\mathrm{a}}=0.478 k_{\mathrm{d}}+0.0229\right)$ was also verified as shown in Figure S6 of Supplementary Material; $k_{\mathrm{a}}$ in Equation (1) could be substituted by $k_{\mathrm{d}}$, making its application easier. In this study, a concept of timescaling IVIVC involving the IR dosage form of sobrerol was proved, and assuming that any modified-release one mimics the model as well.

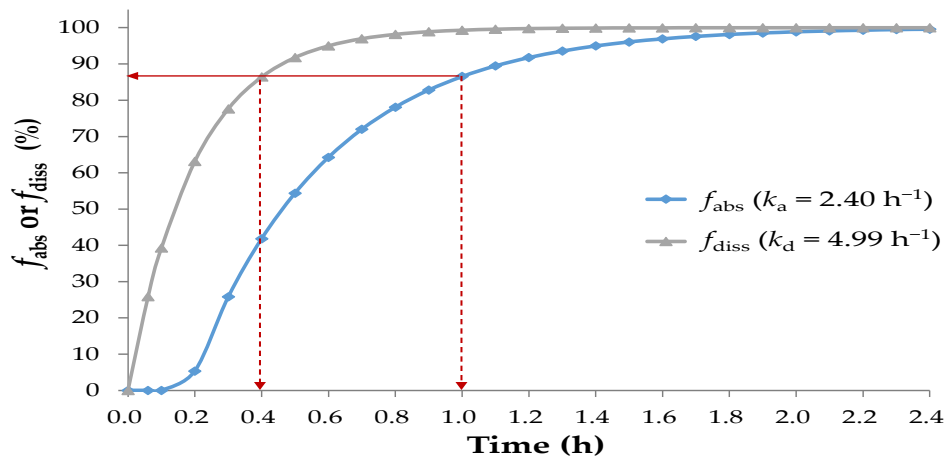

(a)

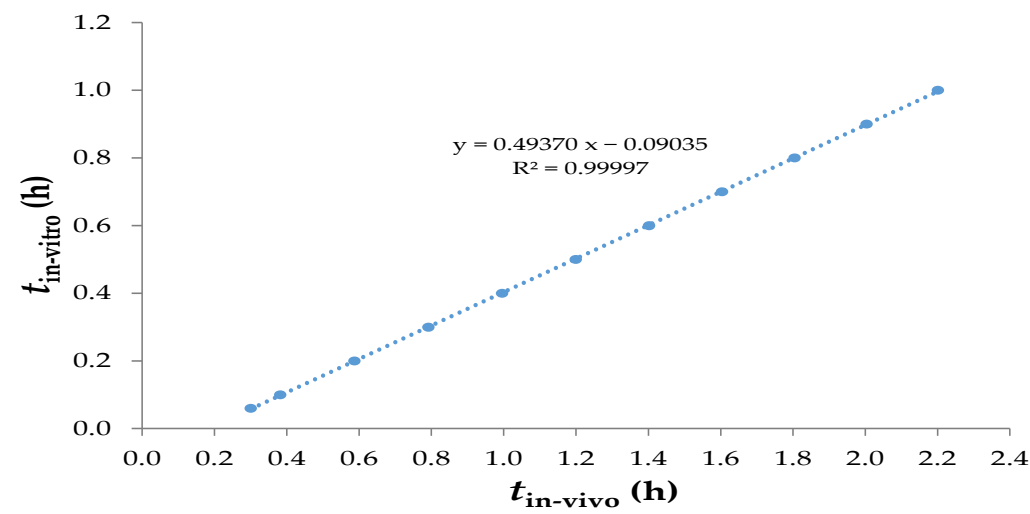

(b)

Figure 3. (a) The in vivo $f_{\text {abs }}$ and in vitro $f_{\text {diss }}$ versus time; (b) A linear regression of $t_{\text {in-vitro }}$ for dissolution versus $t_{\text {in-vivo }}$ for absorption. Indicating that sobrerol had a favorable time-scaling IVIVC. 


\subsubsection{Pharmacokinetic Profiles Derived with Gastrointestinal Transit Time}

Considering the factor of gastrointestinal transit time, the extent of drug absorption is limited. As shown in Figure $4 \mathrm{a}$, the pharmacokinetic profile during the $0-8 \mathrm{~h}$ of absorption followed the oral two-compartment pharmacokinetic model, and that of the absorbed drug during the duration of nonabsorption followed the intravenous two-compartment pharmacokinetic model. As shown in Figure $4 \mathrm{~b}, f_{\text {abs }}$ values decreased with a decrease in $k_{\mathrm{d}}$ values. For example, in the case of the target $f_{\mathrm{abs}}$ is $>95 \%$ by $24 \mathrm{~h}$, the $k_{\mathrm{d}}$ should not be $<0.78 \mathrm{~h}^{-1}$.

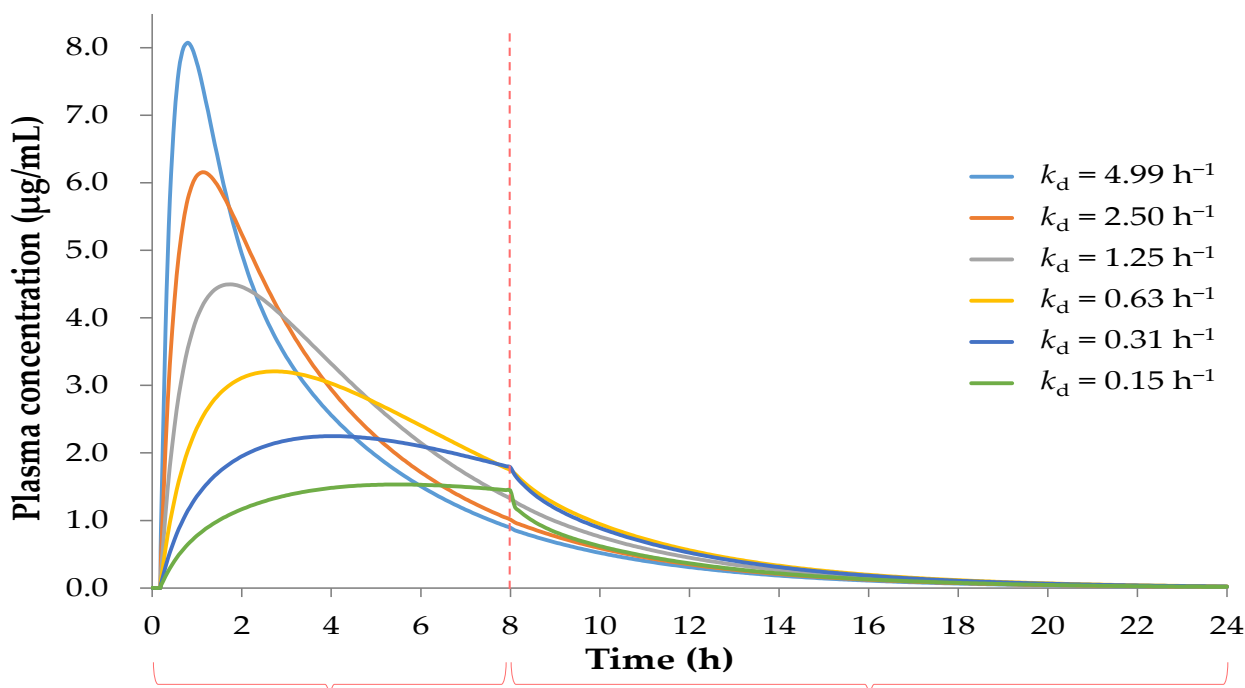

Absorption duration

Nonabsorption duration

(a)

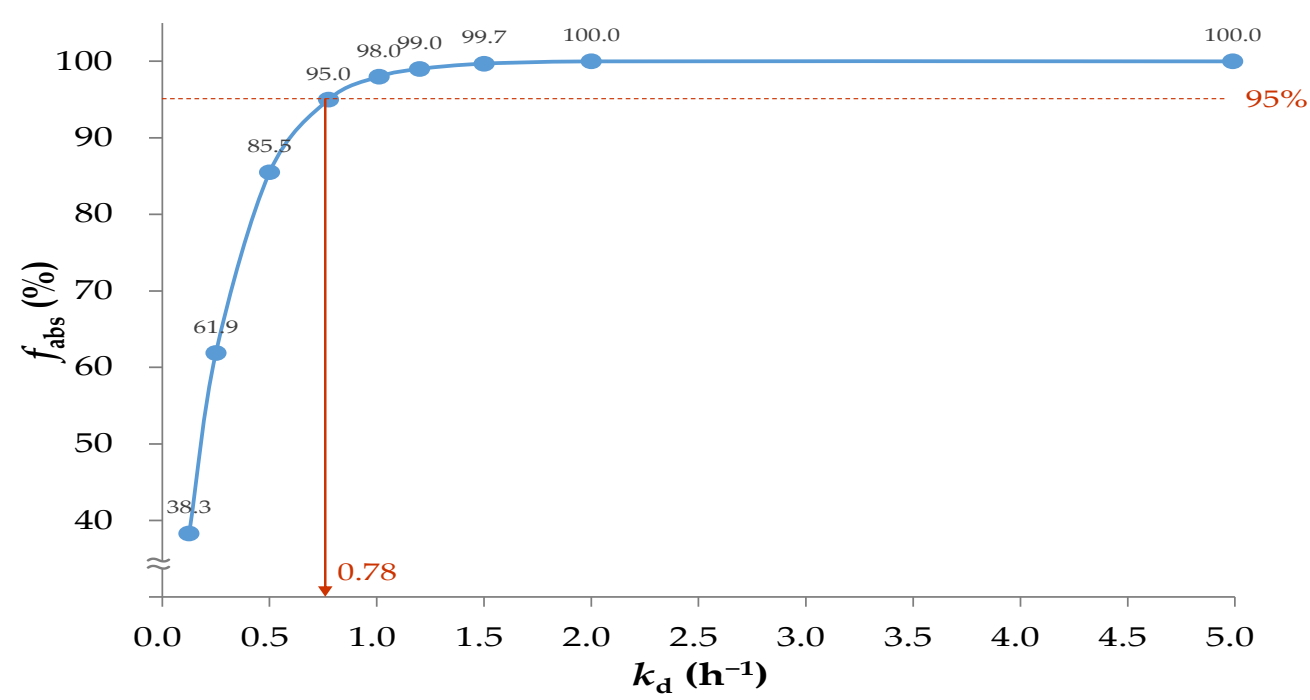

(b)

Figure 4. (a) Simulation of sobrerol oral pharmacokinetic profiles with various $k_{\mathrm{d}}$ values in consideration of gastrointestinal transit time of $8 \mathrm{~h}$; (b) The correlation of $f_{\text {abs }}$ at $24 \mathrm{~h}$ with corresponding $k_{\mathrm{d}}$. In case of the $f_{\mathrm{abs}}$ is $>95 \%$ by $24 \mathrm{~h}$, the $k_{\mathrm{d}}$ should not be $<0.78 \mathrm{~h}^{-1}$.

\subsubsection{Pharmacokinetic Efficacy Index of Sobrerol}

For repurposing sobrerol, the human equivalent doses (HED) and expected symptom inhibition in humans are shown in Table 2, and the related simulated oral pharmacokinetic profiles of administration routes are shown in Figure 5a. No significant therapeutic efficacy 
was observed for an HED of $122 \mathrm{mg}$ (thrice daily) or $146 \mathrm{mg}$ (once daily), and we considered the peak concentration of an HED of $146 \mathrm{mg}$ as the minimum effective concentration (MEC), which is $1.61 \mu \mathrm{g} / \mathrm{mL}$. Symptom inhibitions from high to low were observed with doses of $486 \mathrm{mg}$ (thrice daily), $730 \mathrm{mg}$ (twice daily), and $1459 \mathrm{mg}$ (once daily), which have the same daily dose of $1459 \mathrm{mg}$. The pharmacokinetic efficacy indexes (i.e., $C_{\max }, \mathrm{AUC}$, and $t_{>\mathrm{MEC}}$ ) calculated are also shown in Table 2 , and the pharmacokinetics/pharmacodynamics correlation determined by the linear regression of each index versus the inhibition rate is shown in Figure $5 \mathrm{~b}$. Among them, $t_{>\mathrm{MEC}}$ exhibited the highest correlation with therapeutic efficacy, indicating that this is most likely a time-dependent mechanism of treatment, which explains the observation of higher efficacy at a higher frequency of administration under the same daily dose of the drug. Therefore, we used $t_{>\mathrm{MEC}}$ as the pharmacokinetic efficacy index in the predictive model.

Table 2. Exploring for pharmacokinetics/pharmacodynamics correlation of sobrerol: Human plasma simulation and calculation of pharmacokinetic efficacy indexes including $C_{\max }, \mathrm{AUC}$, and $t_{>\mathrm{MEC}}$.

\begin{tabular}{|c|c|c|c|c|c|c|c|}
\hline \multicolumn{3}{|c|}{ EAE Animal Tests } & \multicolumn{3}{|c|}{ Human Plasma Simulations } & \multirow[b]{2}{*}{$\begin{array}{c}\text { AUC } \\
(\mu \mathrm{g} \cdot \mathrm{h} / \mathrm{mL})\end{array}$} & \multirow[b]{2}{*}{$\begin{array}{c}t_{>\mathrm{MEC}} \\
\text { (h) }\end{array}$} \\
\hline $\begin{array}{c}\text { In Vivo } \\
\text { Dose } \\
\text { (mg/kg) }\end{array}$ & $\begin{array}{l}\text { Frequency } \\
\text { (Daily) }\end{array}$ & $\underset{1,2}{\operatorname{EAE} \text { Score }}$ & $\begin{array}{c}\text { HED }^{3} \\
\text { (mg) }\end{array}$ & $\begin{array}{c}\text { Inhibition } \\
4,5 \\
(\%)\end{array}$ & $\begin{array}{c}C_{\max } \\
(\mu \mathrm{g} / \mathrm{mL})\end{array}$ & & \\
\hline Vehicle & thrice & $4.00 \pm 0.22$ & - & - & - & - & - \\
\hline 25 & thrice & $3.70 \pm 0.15$ & 122 & $\mathrm{~N} / \mathrm{S}$ & 1.48 & 13.59 & 0.0 \\
\hline 30 & once & $3.38 \pm 0.27$ & 146 & $\mathrm{~N} / \mathrm{S}$ & 1.61 & 5.67 & 0.0 \\
\hline 100 & thrice & $\underset{* *}{2.36 \pm 0.27}$ & 486 & 41.00 & 5.91 & 54.13 & 12.8 \\
\hline 150 & twice & $\underset{* *}{2.55 \pm 0.27}$ & 730 & 36.25 & 8.31 & 55.52 & 11.2 \\
\hline 300 & once & $2.78_{* * * *} \pm 0.25$ & 1459 & 30.50 & 16.11 & 56.61 & 8.2 \\
\hline
\end{tabular}

${ }^{1}$ EAE score: EAE signs on the last day after onset (the plateau phase). ${ }^{2 * *} p<0.01$ and ${ }^{* * *} p<0.001$ compared with the vehicle determined using Student's $t$-test. ${ }^{3}$ HED: Human equivalent dose conversed from the EAE animal dose, with a human body weight of $60 \mathrm{~kg} .{ }^{4}$ Inhibition: Decreased ratio of the EAE score compared with the vehicle. ${ }^{5} \mathrm{~N} / \mathrm{S}$ : No significant difference compared with the vehicle.

\subsection{Design of SR Pellets}

In Section 3.2, the characteristics of and the correlation between the pharmacokinetics and pharmacodynamics of sobrerol are discussed, including the pharmacokinetic profile with the lag time, the correlation between the dissolution and absorption of the drug, the gastrointestinal transit time, and the pharmacokinetic efficacy index. Subsequently, the predictive model of oral pharmacokinetic profile for repurposing sobrerol was established. Figure 6 presents the contour plot of the pharmacokinetic efficacy index $t_{>\text {MEC }}$ generated using the predictive model, where the x-axis is $k_{\mathrm{d}}$, the y-axis is the HED by twice daily, and $t_{>\mathrm{MEC}}$ corresponds to the variables of $k_{\mathrm{d}}$ and HED. The original administration route (i.e., $486 \mathrm{mg}$ by thrice daily) exhibits the highest efficacy, with a $t_{>\mathrm{MEC}}$ of $12.8 \mathrm{~h}$ (shown as a red curve), and the SR dosage form is required to exceed the 12.8-h curve to ensure a consistent or higher efficacy of the drug product. The minimum HED is $563 \mathrm{mg}$ (twice daily) at $k_{\mathrm{d}}$ of $0.65 \mathrm{~h}^{-1}$ when compared with the original administration route; this not only reduces the frequency of administration from thrice to twice a day (by $33.3 \%$ ) but also the daily dose from 1459 to $1126 \mathrm{mg}$ (by $22.8 \%$ ), thus considerably improving compliance of administration. 


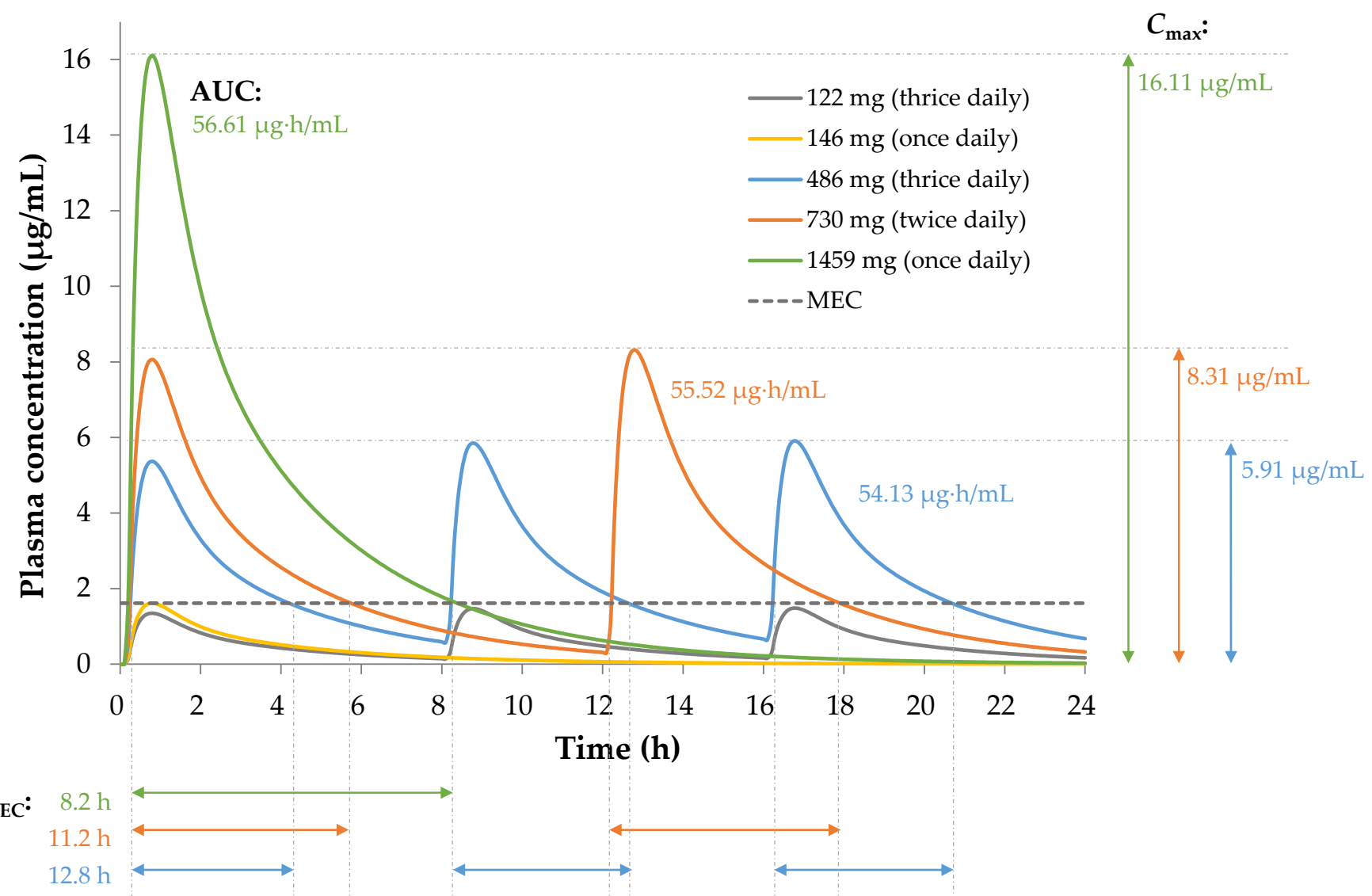

(a)

$1459 \mathrm{mg}$ (once daily) $\quad 730 \mathrm{mg}$ (twice daily) $\quad \square 86 \mathrm{mg}$ (thrice daily)
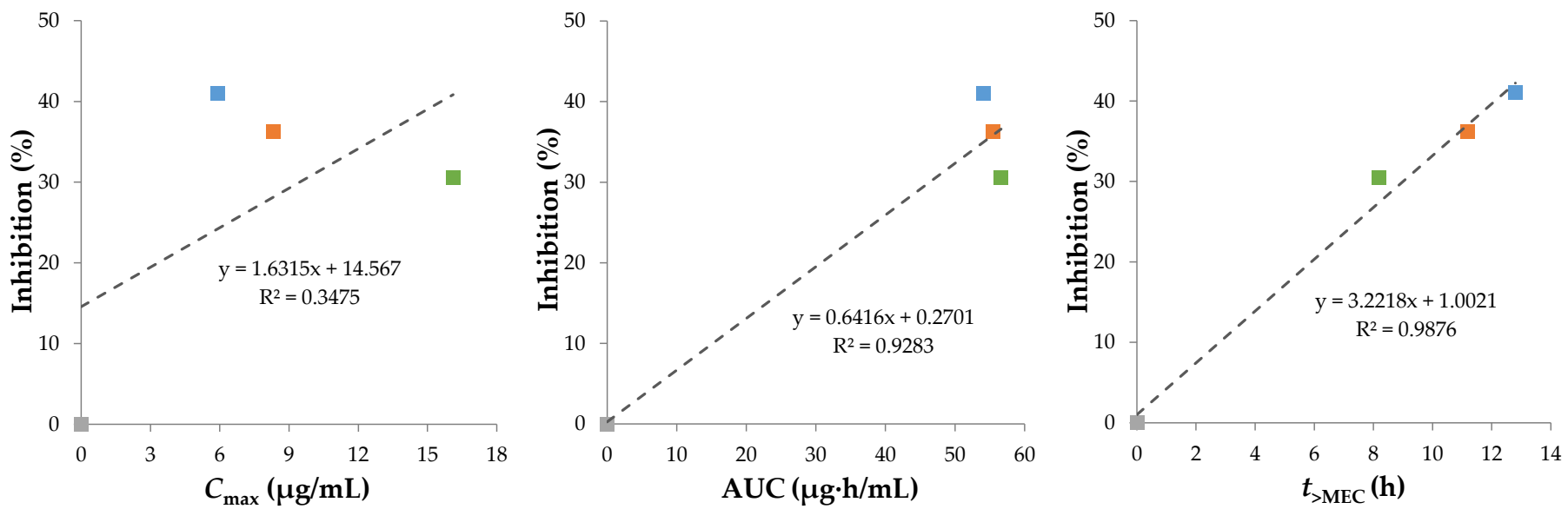

(b)

Figure 5. (a) Simulation for oral pharmacokinetic profiles of sobrerol with the minimum effective concentration (MEC) of $1.61 \mu \mathrm{g} / \mathrm{mL}$, and calculation for pharmacokinetic efficacy indexes $\left(C_{\max }\right.$, AUC, and $t_{>\mathrm{MEC}}$ ) of administration routes with total daily dose of $1459 \mathrm{mg}$ during $24 \mathrm{~h}$. (b) Linear regression of each pharmacokinetic efficacy index versus the inhibition rate, showing that time dependence is responsible for the pharmacokinetics/pharmacodynamics correlation of sobrerol. 


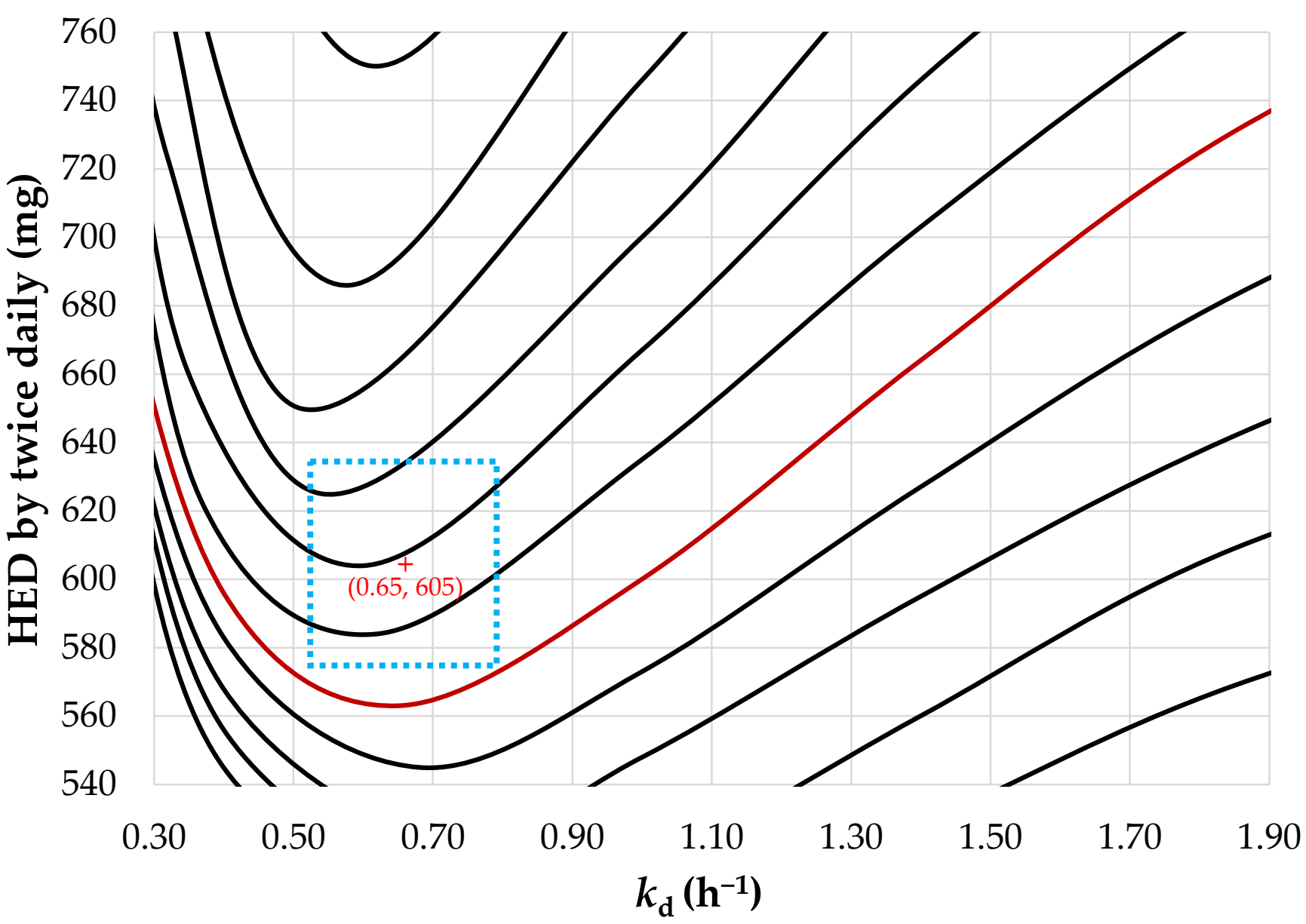

Figure 6. A contour plot of the pharmacokinetic efficacy index $\left(t_{>\mathrm{MEC}}\right)$ corresponding to constants of dissolution $\left(k_{\mathrm{d}}\right)$ and human equivalent dose (HED). The red curve represents the $t_{>\mathrm{MEC}}$ of $12.8 \mathrm{~h}$, which the SR dosage form requires to exceed for a consistent or higher efficacy of the drug product. The blue dotted-line area is as an example as the acceptable specification of the target drug product.

However, the tolerance of drug production must be considered in practice. Deviations may result from various factors, such as coating uniformity or drug loading among batches; these factors can affect the dissolution rate and daily dose of the drug. Assuming that the allowable deviation for coating conditions (i.e., $X_{\mathrm{HPMC} / \mathrm{EC}}$ and $\left.\% W_{\text {coat }}\right)$ is $2 \%$ and that for drug loading is $5 \%$, the boundary conditions to the target $k_{\mathrm{d}}$ (i.e., $0.65 \mathrm{~h}^{-1}$ ) of SR pellets predicted using the DoE are $0.2822 \pm 0.0056$ for $X_{\mathrm{HPMC} / \mathrm{EC}}$ and $9.4560 \pm 0.1891 \%$ for $\% W_{\text {coat }}$, as shown by the green dotted-line area in Figure 7 . In other words, the acceptable specifications of the drug product are tending towards $0.65 \pm 0.13 \mathrm{~h}^{-1}$ for $k_{\mathrm{d}}$ and $605 \pm 30 \mathrm{mg}$ for HED by twice daily, as shown by the blue dotted-line area in Figure 6 .

Finally, we compared the simulated oral pharmacokinetic profiles of IR pellets with a dose of $486 \mathrm{mg}$ thrice daily and target SR pellets with a dose of $605 \mathrm{mg}$ twice daily, and determined that $t_{>\mathrm{MEC}}$ values were 12.8 and $13.6 \mathrm{~h}$, respectively, as shown in Figure 8 . The $t_{>\mathrm{MEC}}$ of SR pellets is longer than that of IR ones, indicating that the SR dosage form is advantageous from the perspectives of both compliance and efficacy improvement. 


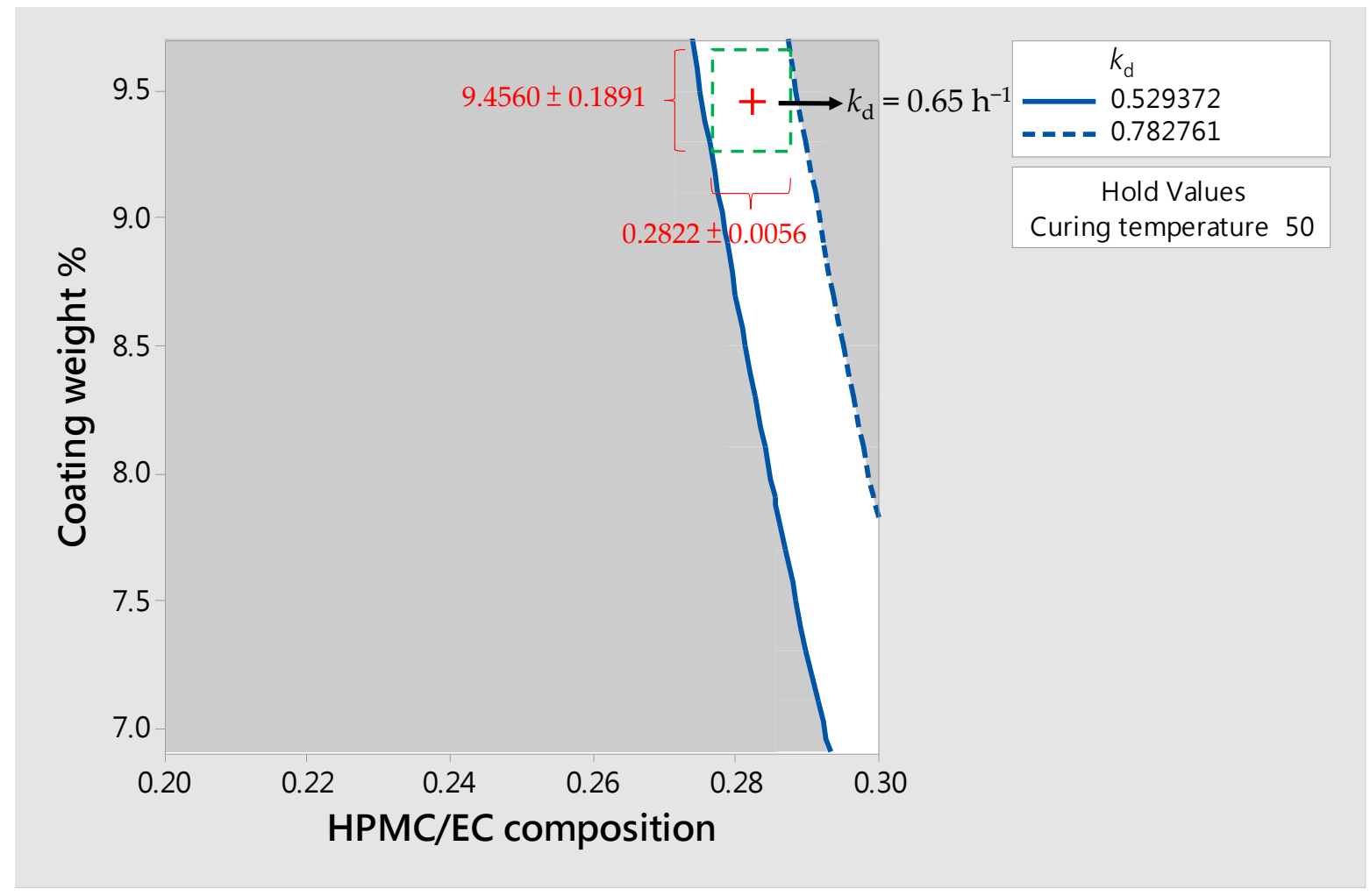

Figure 7. A contour plot of constants of dissolution $\left(k_{\mathrm{d}}\right)$ predicted by the DoE. The boundary conditions to the target $k_{\mathrm{d}}$ of SR pellets is shown by the green dotted-line area, that is, the acceptable specification of $k_{\mathrm{d}}$ is $0.65 \pm 0.13 \mathrm{~h}^{-1}$.

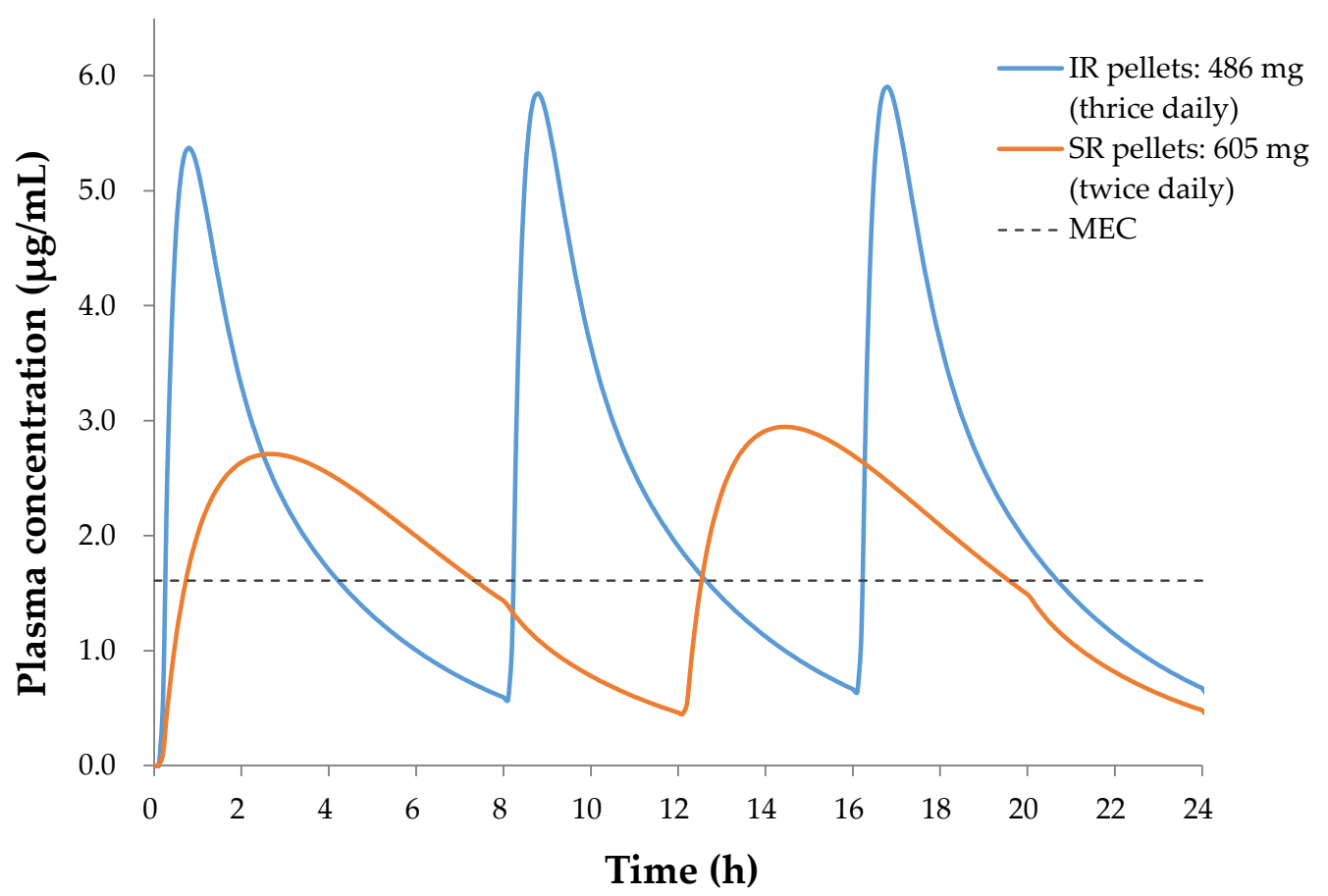

Figure 8. Comparison of pharmacokinetic profiles for the IR pellets ( $4.99 \mathrm{~h}^{-1}$ of $\left.k_{\mathrm{d}}\right)$ and the SR pellets $\left(0.65 \mathrm{~h}^{-1}\right.$ of $\left.k_{\mathrm{d}}\right)$, indicating that the SR pellets is advantageous from the perspectives of both compliance and efficacy improvement. 


\section{Conclusions}

In this study, we successfully developed high-drug-loaded IR and SR pellets of sobrerol. The proposed manufacturing process is simple with high commercial feasibility. $X_{\mathrm{HPMC} / \mathrm{EC}}$ was determined as the main factor for SR pellet release, and the release followed a firstorder model. In addition, a predictive model of oral pharmacokinetic profile based on a time-scaling IVIVC and a time-dependent mechanism for repurposing sobrerol was first established. The predictive model estimated that SR pellets could achieve the goal of improving compliance of administration and therapeutic efficacy in terms of the frequency of administration and dose, that is, taking the drug twice daily and reducing the dose by up to $22.8 \%$. The study also demonstrated a prototype approach of modeling and simulation for rapid design of a drug product, which can reduce the requirement of animal experiments and accelerate research into the clinical status. Future work could further match-up with various pellets of release characteristics to establish an oral multiparticulate system for example, which is highly expandable in medicated application.

Supplementary Materials: The following are available online at https:/ / www.mdpi.com/article/ 10.3390/pharmaceutics14010167/s1, Table S1: BCS classification, Table S2: The formula of SR pellets, Table S3: Kinetic models for drug release study, Table S4: The similarity of the dissolution curves of IR pellets, Table S5: Results for drug release kinetics of SR pellets, Scheme S1: Oral two-compartment model and IVIVC, Figure S1: Results for dissolution of IR pellets, Figure S2: Results for DoE of SR pellets, Figure S3: Fitting plots for SR pellets with various kinetic models, Figure S4: Reconstruction of the sobrerol pharmacokinetic model with lag time, Figure S5: Validation of the time-scaling IVIVC model, Figure S6: The calibration curve of rate constants of in vitro dissolution and in vivo absorption.

Author Contributions: Conceptualization, C.-H.L.; methodology, C.-H.L. and Y.-F.H.; software, C.-H.L. and Y.-F.H.; validation, C.-H.L., Y.-F.H., and I.-M.C.; formal analysis, C.-H.L. and Y.-F.H.; investigation, C.-H.L. and Y.-F.H.; resources, C.-H.L. and I.-M.C.; data curation, C.-H.L. and Y.-F.H.; writing-original draft preparation, C.-H.L.; writing-review and editing, C.-H.L., Y.-F.H., and I.M.C.; visualization, C.-H.L.; supervision, C.-H.L. and I.-M.C. All authors have read and agreed to the published version of the manuscript.

Funding: This research was supported by the Department of Industrial Technology of the Ministry of Economic Affairs, Taiwan under Grant No. 107-EC-17-A-22-0318.

Institutional Review Board Statement: Not applicable.

Informed Consent Statement: Not applicable.

Data Availability Statement: Not applicable.

Acknowledgments: The authors thank De-Hao Tsai at the National Tsing Hua University for technical support and helpful discussions.

Conflicts of Interest: The authors declare no conflict of interest.

\section{References}

1. Braga, P.C.; Allegra, L.; Bossi, R.; Scuri, R.; Castiglioni, C.L.; Romandini, S. Review on sobrerol as a muco-modifying drug: Experimental data and clinical findings in hypersecretory bronchopulmonary diseases. Int. J. Clin. Pharmacol. Res. 1987, 7, 381-400.

2. Castiglioni, C.L.; Gramolini, C. Effect of long-term treatment with sobrerol on the exacerbations of chronic bronchitis. Respiration 1986, 50, 202-217. [CrossRef] [PubMed]

3. Bellussi, L.; Manini, G.; Buccella, M.; Cacchi, R. Evaluation of the efficacy and safety of sobrerol granules in patients suffering from chronic rhinosinusitis. J. Int. Med. Res. 1990, 18, 454-459. [CrossRef]

4. Scaglione, F.; Petrini, O. Mucoactive Agents in the Therapy of Upper Respiratory Airways Infections: Fair to Describe Them Just as Mucoactive? Clin. Med. Insights Ear Nose Throat 2019, 12, 1179550618821930. [CrossRef] [PubMed]

5. Huang, K.K.; Pan, I.H.; Wen, S.F.; Lin, M.N.; Lu, I.H.; Kuo, Z.K.; Lu, C.H.; Lee, T.C.; Yang, Y.Y.; Liaw, J.H. Method for Treating an Autoimmune Neurological Disease and/or Neurodegenerative Disease and Pharmaceutical Formulations for a Liquid Dosage form and a Controlled Release Dosage form. U.S. Patent Pub. No.: US20190000776A1, 3 January 2019.

6. $\mathrm{Ng}$, X.; Sadeghian, M.; Heales, S.; Hargreaves, I.P. Assessment of Mitochondrial Dysfunction in Experimental Autoimmune Encephalomyelitis (EAE) Models of Multiple Sclerosis. Int. J. Mol. Sci. 2019, 20, 4975. [CrossRef] [PubMed] 
7. Stavropoulos, F.; Georgiou, E.; Sargiannidou, I.; Kleopa, K.A. Dysregulation of Blood-Brain Barrier and Exacerbated Inflammatory Response in Cx47-Deficient Mice after Induction of EAE. Pharmaceuticals 2021, 14, 621. [CrossRef] [PubMed]

8. Serra, A.; Fox, R.J. Dimethyl fumarate for relapsing MS. Neurol Clin. Pract. 2013, 3, 249-253. [CrossRef] [PubMed]

9. Spencer, C.M.; Crabtree-Hartman, E.C.; Lehmann-Horn, K.; Cree, B.A.; Zamvil, S.S. Reduction of CD8(+) T lymphocytes in multiple sclerosis patients treated with dimethyl fumarate. Neurol Neuroimmunol. Neuroinflamm. 2015, 2, e76. [CrossRef]

10. Palugan, L.; Cerea, M.; Zema, L.; Gazzaniga, A.; Maroni, A. Coated pellets for oral colon delivery. J. Drug Deliv. Sci. Technol. 2015, 25, 1-15. [CrossRef]

11. Abdul, S.; Chandewar, A.V.; Jaiswal, S.B. A flexible technology for modified-release drugs: Multiple-unit pellet system (MUPS). J. Control Release 2010, 147, 2-16. [CrossRef] [PubMed]

12. Gao, Y.; Jin, X.; Sun, Y.; Xu, F.; Zhang, M. Production and investigation of sustained berberine pellet drug release system. Adv. Powder Technol. 2018, 29, 682-691. [CrossRef]

13. Lopez, F.L.; Bowles, A.; Gul, M.O.; Clapham, D.; Ernest, T.B.; Tuleu, C. Effect of formulation variables on oral grittiness and preferences of multiparticulate formulations in adult volunteers. Eur. J. Pharm. Sci. 2016, 92, 156-162. [CrossRef] [PubMed]

14. Wadher, K.J.; Kakde, R.B.; Umekar, M.J. Study on sustained-release metformin hydrochloride from matrix tablet: Influence of hydrophilic polymers and in vitro evaluation. Int. J. Pharm. Investig. 2011, 1, 157-163. [CrossRef] [PubMed]

15. Albanez, R.; Nitz, M.; Taranto, O.P. Enteric coating process of diclofenac sodium pellets in a fluid bed coater with a wurster insert: Influence of process variables on coating performance and release profile. Adv. Powder Technol. 2013, 24, 659-666. [CrossRef]

16. Dey, N.; Majumdar, S.; Rao, M. Multiparticulate drug delivery systems for controlled release. Trop. J. Pharm. Res. 2008, 7, 1067-1075. [CrossRef]

17. Kushwah, V.; Arora, S.; Tamás Katona, M.; Modhave, D.; Fröhlich, E.; Paudel, A. On Absorption Modeling and Food Effect Prediction of Rivaroxaban, a BCS II Drug Orally Administered as an Immediate-Release Tablet. Pharmaceutics 2021, 13, 283. [CrossRef] [PubMed]

18. Chavda, V.P.; Shah, D.; Tandel, H.; Soniwala, M. In Vitro-In Vivo Correlation (IVIVC): A Strategic Tool in Drug Product Development. J. Drug Formul. Dev. Prod. 2016, 3, 31-54.

19. Sipos, B.; Szabó-Révész, P.; Csóka, I.; Pallagi, E.; Dobó, D.G.; Bélteky, P.; Kónya, Z.; Deák, Á.; Janovák, L.; Katona, G. Quality by Design Based Formulation Study of Meloxicam-Loaded Polymeric Micelles for Intranasal Administration. Pharmaceutics 2020, 12, 697. [CrossRef] [PubMed]

20. Braga, P.C.; De Angelis, L.; Bossi, R.; Scaglione, F.; Scarpazza, G.; Allegra, L.; Fraschini, F. Pharmacokinetics of Sobrerol in chronic bronchitis. Comparison of serum and bronchial mucus levels. Eur. J. Clin. Pharm. 1983, 24, 209-215. [CrossRef]

21. Yalkowsky, S.H.; He, Y.; Jain, P. Handbook of Aqueous Solubility Data, 2nd ed.; CRC Press: Boca Raton, FL, USA, 2016 ; p. 731.

22. Kambayashi, A.; Blume, H.; Dressman, J.B. Predicting the oral pharmacokinetic profiles of multiple-unit (pellet) dosage forms using a modeling and simulation approach coupled with biorelevant dissolution testing: Case example diclofenac sodium. Eur. J. Pharm. Biopharm. 2014, 87, 236-243. [CrossRef]

23. Vlachou, M.; Karalis, V. An In Vitro-In Vivo Simulation Approach for the Prediction of Bioequivalence. Materials 2021, 14, 555. [CrossRef] [PubMed]

24. Muley, S.; Nandgude, T.; Poddar, S. Extrusion-spheronization a promising pelletization technique: In-depth review. Asian J. Pharm. Sci. 2016, 11, 684-699. [CrossRef]

25. Broesder, A.; Bircan, S.Y.; de Waard, A.B.; Eissens, A.C.; Frijlink, H.W.; Hinrichs, W.L.J. Formulation and In Vitro Evaluation of Pellets Containing Sulfasalazine and Caffeine to Verify Ileo-Colonic Drug Delivery. Pharmaceutics 2021, 13, 1985. [CrossRef] [PubMed]

26. KFDA. The Korean Pharmacopoeia, 11th ed.; Korea Food and Drug Administration: Seoul, Korea, 2014; pp. 758-759.

27. Jantratid, E.; De Maio, V.; Ronda, E.; Mattavelli, V.; Vertzoni, M.; Dressman, J.B. Application of biorelevant dissolution tests to the prediction of in vivo performance of diclofenac sodium from an oral modified-release pellet dosage form. Eur. J. Pharm. Sci. 2009, 37, 434-441. [CrossRef] [PubMed]

28. Patel, N.; Lalwani, D.; Gollmer, S.; Injeti, E.; Sari, Y.; Nesamony, J. Development and evaluation of a calcium alginate based oral ceftriaxone sodium formulation. Prog. Biomater. 2016, 5, 117-133. [CrossRef]

29. Puhl, D.L.; Funnell, J.L.; D'Amato, A.R.; Bao, J.; Zagorevski, D.V.; Pressman, Y.; Morone, D.; Haggerty, A.E.; Oudega, M.; Gilbert, R.J. Aligned Fingolimod-Releasing Electrospun Fibers Increase Dorsal Root Ganglia Neurite Extension and Decrease Schwann Cell Expression of Promyelinating Factors. Front. Bioeng. Biotechnol. 2020, 8, 937. [CrossRef]

30. Venkatesh, G.M.; Stevens, P.J.; Lai, J.W. Development of orally disintegrating tablets comprising controlled-release multiparticulate beads. Drug Dev. Ind. Pharm. 2012, 38, 1428-1440. [CrossRef]

31. Sánchez-Dengra, B.; González-García, I.; González-Álvarez, M.; González-Álvarez, I.; Bermejo, M. Two-step in vitro-in vivo correlations: Deconvolution and convolution methods, which one gives the best predictability? Comparison with one-step approach. Eur. J. Pharm. Biopharm. 2021, 158, 185-197. [CrossRef]

32. Figueroa-Campos, A.; Sánchez-Dengra, B.; Merino, V.; Dahan, A.; González-Álvarez, I.; García-Arieta, A.; González-Álvarez, M.; Bermejo, M. Candesartan Cilexetil In Vitro-In Vivo Correlation: Predictive Dissolution as a Development Tool. Pharmaceutics 2020, 12, 633. [CrossRef]

33. Qureshi, S.A. In vitro-in vivo correlation (ivivc) and determining drug concentrations in blood from dissolution testing-a simple and practical approach. Open Drug Deliv. J. 2010, 4, 38-47. [CrossRef] 
34. Hua, S. Advances in oral drug delivery for regional targeting in the gastrointestinal tract-Influence of physiological, pathophysiological and pharmaceutical factors. Front. Pharm. 2020, 11, 524. [CrossRef]

35. Bounda, G.A.; Zhou, W.; Wang, D.D.; Yu, F. Rhein Elicits In Vitro Cytotoxicity in Primary Human Liver HL-7702 Cells by Inducing Apoptosis through Mitochondria-Mediated Pathway. Evid. Based Complement. Altern. Med. 2015, 2015, 329831. [CrossRef]

36. Gupta, P.K. Illustrated Toxicology: With Study Questions, 1st ed.; Academic Press: Cambridge, MA, USA, 2018 ; p. 86. 